This article was downloaded by: [184.57.72.251]

On: 03 J une 2013, At: 01:17

Publisher: Routledge

Informa Ltd Registered in England and Wales Registered Number: 1072954 Registered office: Mortimer House, 37-41 Mortimer Street, London W1T 3J H, UK

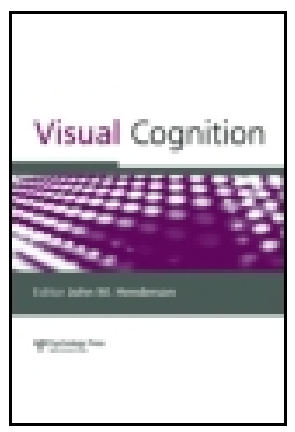

\title{
Visual Cognition
}

Publication details, including instructions for authors and subscription information:

http:/ / www. tandfonline.com/loi/ pvis20

\section{Gaze and informativeness during category learning: Evidence for an inverse relation}

\author{
Ronaldo Vigo $^{a}$, Derek E. Zeigler ${ }^{a} \&$ Phillip A. Halsey ${ }^{a}$ \\ ${ }^{a}$ Center for the Advancement of Cognitive Science, \\ Psychology Department, Ohio University , Athens, \\ $\mathrm{OH}$, USA \\ Published online: 03 J un 2013.
}

To cite this article: Ronaldo Vigo, Derek E. Zeigler \& Phillip A. Halsey (2013): Gaze and informativeness during category learning: Evidence for an inverse relation, Visual Cognition, DOI: 10.1080/ 13506285.2013.800931

To link to this article: http:/ / dx. doi.org/ 10.1080/ 13506285.2013.800931

\section{PLEASE SCROLL DOWN FOR ARTICLE}

Full terms and conditions of use: http://www.tandfonline.com/page/termsand-conditions

This article may be used for research, teaching, and private study purposes. Any substantial or systematic reproduction, redistribution, reselling, loan, sublicensing, systematic supply, or distribution in any form to anyone is expressly forbidden.

The publisher does not give any warranty express or implied or make any representation that the contents will be complete or accurate or up to date. The accuracy of any instructions, formulae, and drug doses should be independently verified with primary sources. The publisher shall not be liable for any loss, actions, claims, proceedings, demand, or costs or damages whatsoever or howsoever caused arising directly or indirectly in connection with or arising out of the use of this material. 


\title{
Gaze and informativeness during category learning: Evidence for an inverse relation
}

\author{
Ronaldo Vigo, Derek E. Zeigler, and Phillip A. Halsey \\ Center for the Advancement of Cognitive Science, Psychology \\ Department, Ohio University, Athens, OH, USA
}

\begin{abstract}
In what follows, we explore the general relationship between eye gaze during a category learning task and the information conveyed by each member of the learned category. To understand the nature of this relationship empirically, we used eye tracking during a novel object classification paradigm. Results suggest that the average fixation time per object during learning is inversely proportional to the amount of information that object conveys about its category. This inverse relationship may seem counterintuitive; however, objects that have a highinformation value are inherently more representative of their category. Therefore, their generality captures the essence of the category structure relative to less representative objects. As such, it takes relatively less time to process these objects than their less informative companions. We use a general information measure referred to as representational information theory (Vigo, 2011a, 2013a) to articulate and interpret the results from our experiment and compare its predictions to those of three models of prototypicality.
\end{abstract}

Keywords: Category learning; Eye movements; Math modelling; Object-based attention; Representational information.

Whether a person is learning a complex programming language or deciding which pair of shoes to buy, the amount of time devoted to any particular item may be influenced by the amount of information that it conveys and the context in which it is presented. In other words, the amount of time spent looking at a given stimulus is likely a function of its level of informativeness.

Please address all correspondence to Ronaldo Vigo, Asst. Professor of Mathematical \& Computational Cognitive Science, Center for the Advancement of Cognitive Science, Ohio University, Athens, OH 45701, USA. E-mail: vigo@ohio.edu

The authors would like to thank Mark Gould for his invaluable help with data management as well as Mikayla Barcus, Charles Doan, Basawaraj, Yu Zhang, and Jinling Zhao for their helpful comments on an earlier version of this manuscript. 
This link between information and fixation duration conforms to our intuitions about what influences scene inspection (Henderson \& Hollingworth, 1999; Rayner, Smith, Malcolm, \& Henderson, 2009). Indeed, we presumably fixate on aspects of a scene because these aspects are in some way informative and fixation duration depends on their degree of overall informativeness. For example, we glance at a roadside sign because we believe that it may hold information regarding our destination and fixate longer if it proves informative but search elsewhere if the information is irrelevant. In this paper, we discuss visual fixations during information search and report how fixation durations during scene inspection align with a particular operationalization and general measure of information.

Eye-tracking technology provides a powerful tool applicable to many domains of vision research (see Mele \& Federici, 2012). Due to its overt measurement of gaze, researchers are able to obtain accurate spatiotemporal data in real time, making it particularly useful when exploring the link between information and fixations. However, results are often restricted to narrow subdomains making it difficult to speak generally about the relationship between fixation time and information. Nevertheless, because numerous studies have revealed that saccadic eye movements often reflect cognitive processes (Kowler, 1991; Viviani, 1991; Yarbus, 1967), eye tracking has been instrumental in research concerning visual search (e.g., Liversedge, \& Findlay, 2000), reading (e.g., Rayner, 1998), and a range of other cognitive tasks (see Hayhoe \& Ballard, 2005). Notwithstanding, evidence from the eye-tracking literature also suggests that eye gaze often has a "mind of its own" because it conforms to the automatic motor propensities inherited from, and fine-tuned by, the functional evolution of the human visual system (Martinez-Conde, Macknik, \& Hubel, 2004; Rolfs, 2009). This suggests the link between mental processes and subsequent eye movements may not correspond in all situations.

Likewise, research on human classification performance suggests a possible link between gaze and higher order information processing. These theories typically interpret stimulus features as the primary source of information and, consequently, their focus has been on determining fixations on the stimulus dimensions. Specifically, researchers have presumed that humans learn to fixate on those dimensions diagnostic of category membership (e.g., Medin \& Schaffer, 1978; Shepard, Hovland, \& Jenkins, 1961).

This general assumption has been validated by a previous eye-tracking investigation of category learning which used a trial-by-trial learning task in which attention was found to be optimally distributed between stimulus dimensions (Rehder \& Hoffman, 2005). Indeed, when features are spatially separated, participants adjust their fixations in favour of informative features during category learning. This bias for informative features presumably optimizes subsequent classification performance. 
Whereas previous studies have been instrumental in demonstrating fixation biases towards features diagnostic of category membership, the current study examines how the informativeness of an object as a whole influences visual fixation times. Our focus on objects as a whole is important because the dimensions of most real-world objects are not spatially separated, but instead are composed of features that collectively define the informativeness of an object as a Gestalt judgement. In other words, information about an object is typically implicitly elicited rather than actively, explicitly, and meticulously searched for in space. Additionally, psychophysical studies show that humans can get the essence of a scene within $100 \mathrm{~ms}$ of exposure but it takes much longer to process the finegrained details of individual objects (Rayner et al., 2009).

Accordingly, whereas previous studies demonstrate a fixation bias towards features diagnostic of category membership, the current study examines how the informativeness of an object as a whole influences visual search. In accordance with the level of information processing based on whole objects, we use a novel classification task as a means to examine how the structure of environmental stimuli influences gaze. We propose that certain key constructs underlying prototype models of categorization may be useful in accounting for fixation times at the whole-object level of processing during category learning. In addition to these key constructs, a general measure of information proposed by Vigo (2011a) is included to articulate and interpret the results. Next, we describe these constructs and how they may be used to account for eye movement during category learning.

Representational Information Theory, or RIT (Vigo, 2011a), provides a possible solution to the problem of how humans judge the degree to which an object is informative depending on the context within which it occurs. In RIT, a new general way of characterizing information is proposed that is based on five principles: (1) that humans communicate via concepts or, in other words, mental representations of categories of objects (where a category is simply a set of objects that are related in some way); (2) concepts are the mediators of information; (3) concepts are relationships between qualitative objects in the environment that are defined dimensionally; (4) the degree of coherence or Gestalt homogeneity of a category (i.e., to what overall extent its objects are indistinguishable) as well as its cardinality (i.e., size) determine the learnability or complexity of its associated concept; and (5) information is the rate of change of that complexity (and more specifically, for discrete functions, the proportional change of that complexity). The first three principles are frequently adopted by researchers in the field of human concept learning (Bourne, 1966; Estes, 1994; Garner, 1974; Vigo, 2009a, 2009b, 2009c), and the fourth and fifth form the basis of the theory proposed by Vigo (2009a, 2011b, 2013b). 
Combined, these principles support the proposition that the amount of information conveyed by a subset of instances of a dimensionally defined category is the rate of change in the structural complexity of the category whenever the instances are removed from it (Vigo, 2011a). The rate of change is the percent increase or decrease in the structural complexity of the category. The structural complexity of the category is measured by the exponential categorical invariance model (ECIM; Vigo, 2009a, 2011b).

Details as to how to compute the information of the objects of a categorical stimulus with the same structure as that of the fifth categorical stimulus in Figure 1 are given in the Technical Appendix.

Thus, with RIT, we are able to assign an information value to each object of any category structure. Negative information values indicate a decrease in the complexity of the category when a given object is removed whereas positive values indicate an increase in complexity with the object's removal from the set. Thus, with RIT one can measure not only the relative magnitude of representational information (rate of change in complexity) but also the quality of the information as represented by the direction of the rate of change (in terms of negative and positive values). This ability to

A
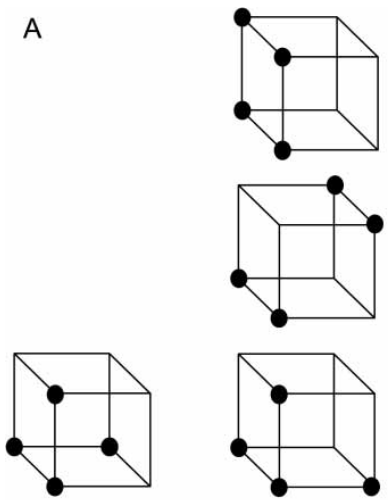
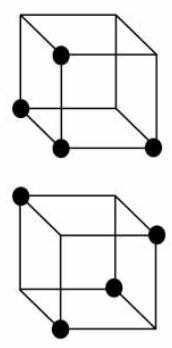

B

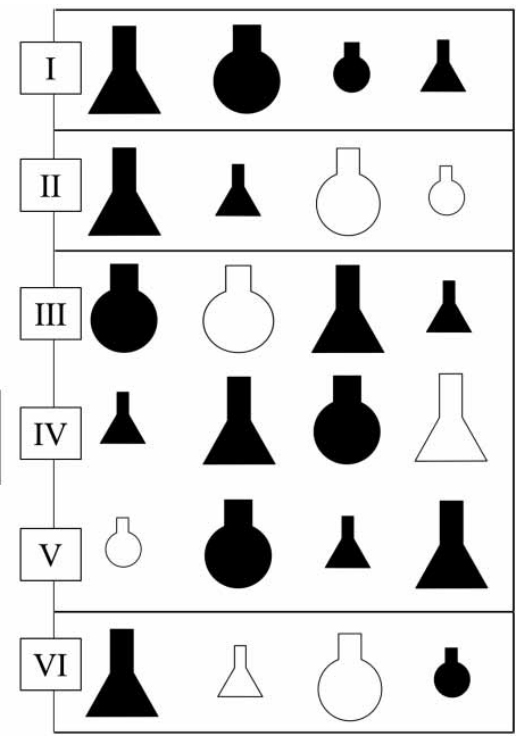

Figure 1. Examples of category structures used in the present study: These consist of four objects defined over three dimensions (in this example: colour, shape, and size). (A) The stimuli of each type denoted by the corners of a cube where the sides of the cube represent dimensions. Corners with circles represent positive examples, whereas empty corners are negative examples of the category. (B) Examples of category instances for each type. Try learning the objects in each type. Which objects might you need to spend more time looking at in order to learn the category? 
measure the quantity and quality of information is unique among information measures. Furthermore, the idea of linking the change in the complexity of a category structure (when some of its elements are removed) to information content addresses the problem of determining the role that context plays on the amount of information humans attribute to each object of a category.

An alternative account of eye movements during category learning might be provided by theories of concept learning rather than the information theoretic account just described. Particularly relevant explanations of the data reported here are described by the three ways of measuring prototypicality. We intend to compare the predictions made by RIT and by these three prototypicality measures against the data reported in the current experiment. The first approach, suggested by Rosch (1978), we refer to as the Feature Frequency Prototype Model (FFPM). Using this classic prototype account, the degree of prototypically of each object is computed by adding, across all three dimensions, the number of objects in the category that share the same dimensional value (i.e., feature) with the object. Moreover, we computed the degree of prototypicality of each object in each category by using the similarity measure at the core of the multiplicative prototype model (MPM; Estes, 1986; Nosofsky \& Zaki, 2002). The MPM uses Shepard's similarity measure (Shepard, 1974, 1987) to compute the similarity between each object and the category's prototype. There are two notions of prototypicality used by two different versions of the MPM. Estes' version of the MPM (1986) computes the prototype of a category by taking the average value, per dimension, of all the objects in the category. On the other hand, Nosofsky and Zaki (2002) determine the prototype of the category by generating the object that consists of the most frequently occurring dimensional values (i.e., features) among the objects of the category. We shall refer to these two distinct approaches to measuring prototypicality as MPMP-1 (Multiplicative Prototype Model Prototypicality 1) and MPMP-2 (Multiplicative Prototype Model Prototypicality 2), respectively. The reader is referred to Nosofsky and Zaki for a detailed explanation of these approaches, and to the appendix of Vigo and Basawaraj (2013) for a summary discussion and tutorial on how to compute the three aforementioned models of prototypicality.

\section{CURRENT STUDY}

Participants were instructed to learn a category or set of four objects during a learning phase. Immediately after the learning phase, objects were classified as members or nonmembers of the learned category. All four members of the category were presented simultaneously, making whole 
objects the locus of fixation. Eye movements were recorded while participants viewed the objects during the learning phase. The categories of objects presented to participants reflected the category structure types associated with four objects defined over three binary dimensions; these were studied extensively by previous researchers (e.g., Shepard et al., 1961). Six types of category structures are possible from stimuli with three binary dimensions and four objects (see Figure 1). These structures are generalizable to many real-world stimuli but their instances remain moderately difficult to learn, making them exceptional for studying conceptual behaviour. Their degree of learning difficulty, as determined by the proportion of correct object classifications, differs among these category structures. This learning difficulty ordering is robust and well replicated empirically (Kruschke, 1992; Love, Medin, \& Gureckis, 2004; Nosofsky, Palmeri, \& McKinley, 1994; Vigo, 2011b, 2013b) and is generally interpreted as revealing key psychological principles underlying human conceptual behaviour.

The experimental paradigm differs from previous categorization tasks in the way that: (1) the category is fully shown during the learning phase, (2) nonmembers of the category are not shown during the learning phase, and (3) corrective feedback is not provided during the classification phase. Instead, in an initial learning phase, a participant learns the category conceptually by studying its four members. Learning is then assessed by a subsequent classification task during which the members and nonmembers of the category are presented one at a time and at random. Participants then provide "yes" and "no" responses (by clicking a mouse button) indicating which objects belong and do not belong in the category. In contrast, previous studies use a dynamic learning task that presents the members and nonmembers of the category at random on a trial-by-trial, object-by-object basis with corrective feedback after each classification decision (Nosofsky, Gluck, Palmeri, Mckinley, \& Glauthier, 1994). To determine how both spatial locations and length of fixations are influenced by these six structures presented in the learning phase, we obtained fixation times and saccadic activity while participants learned these categories.

We found that participants fixated longer on objects of the category that have a low information value as measured by RIT. This inverse relationship may seem counterintuitive; however, this tendency may be adaptive during an information search process that is highly influenced by context. For instance, objects that have a high information value are inherently more representative of the category; thus, the generality of these objects easily captures the essence of the category structure relative to other less representative objects. If this is the case, it would take little time to process category membership for informative objects and more time to process less informative, less representative objects in the category. The smaller amount of average fixation time for the most informative objects may be a reflec- 
tion of this process of learning such objects at a quicker rate. Regarding models of typicality, we show that individuals fixate longer on objects that are less prototypical of their category. This finding is consistent with our RIT prediction in the way that objects that are less informative are inherently less prototypical. Thus, in general, we propose an inverse quantitative relation between proportion of dwell time and the degree of informativeness (as measured in RIT) or, respectively, the degree of prototypicality (as measured in the aforementioned prototype theories) of the objects in a category structure. We refer to the "inverse of information" relation as the IMF (information model of fixation) and to the "inverse of prototypicality" relation as the PMF (prototype model of fixation). Clearly, there is a PMF model corresponding to each of the aforementioned ways of computing degree of prototypicality.

\section{METHOD}

\section{Participants}

The participants were 40 undergraduates from Ohio University who received course credit for their participation. All had normal or corrected-to-normal vision.

\section{Stimuli and procedure}

The categories presented to participants consisted of flasks that varied on three binary features: size (large or small), shape (circular or triangular), and colour (black or white). Figure 1 presents an example of the categorical stimuli used in the task. The flasks were arranged in a two by two matrix so as to reduce likely biases towards particular areas of the display. The locations of the four stimuli within this two by two matrix were randomized across trials. Participants were told that each category of four flasks represented the preferences of a bottle collector and that their task was to learn the flasks that the bottle collector likes.

After $20 \mathrm{~s}$ of studying the four flasks, individual flasks appeared on the screen and participants were instructed to identify whether the flask was or was not part of the category liked by the bottle collector.

To do so, participants were instructed to respond via a mouse click: left for yes (the flask is liked) and right for no (the flask is not liked) as objects appeared for classification. Each block was comprised of eight flasks (four members and four nonmembers) that were serially presented and categorized by participants. After responses had been given for these eight flasks, a fixation cross appeared in the centre of the screen and the next category block was shown. Each participant was presented with six instances of the 
six category structures resulting in a 36 block session lasting approximately 30-40 minutes. An "instance" of a structure type refers to a categorical stimulus that is defined by the structure type (or reflects its structure). Such instances defined by the same structure type are structurally equivalent but differ in their dimensional assignments. Specifically, there are 70 distinct categorical stimuli defined by the six structure types in the 3[4] family of structure types (where the number three in the 3[4] notation stands for the number of dimensions that the structure type specifies and the number four stands for the number of category members) and are distributed as follows: [I (6); II (6); III (24); IV (8); V (24); VI (2)]. Six instances of each type were randomly chosen from this pool of instances. The same randomly chosen instances were given to each participant in order to compare gaze per type between participants (the two instances of type VI were shown to participants three times each resulting in six trials). These instances, as well as the eight objects presented for categorization, were presented to participants randomly according to a counterbalanced design throughout the experimental session and classification performance was obtained for each participant.

\section{Equipment}

An EyeLink 2000 eye-tracker by SR Research ${ }^{\mathrm{TM}}$ with a sampling rate of 2 $\mathrm{kHz}$, recorded movements of the participant's left eye throughout the entire experimental session. After a practice trial where participants were presented two instances of the task, participants were fitted to the head mount which was adjusted for comfort. The participants were then calibrated to the eyetracker by fixating on a small circle presented in different areas of the monitor. This procedure allows for drift corrections and small calibration adjustments to be made for each participant. The EyeLink software recognizes average fixation errors of less than $\sim 1 \mathrm{~mm}\left(\sim 0.08^{\circ}\right.$ visual angle) from the target as acceptable for eye tracking to proceed. Only participants with calibrations deemed "good" by the EyeLink software were included in the analysis (40 of 47 participants received "good" calibrations; data from the additional seven participants was discarded). The average error rate across participants included in the study was $0.43 \mathrm{~mm}$ with a range of $0.25 \mathrm{~mm}$ to $0.79 \mathrm{~mm}\left(0.02^{\circ}\right.$ to $0.06^{\circ}$ visual angle $)$.

The objects were presented on a light grey background (RGB: 128, 128, 128) on a 17-inch Dell computer monitor. The head mount was placed 29 inches $(73.55 \mathrm{~cm})$ away from the monitor. On account of size being one of the three diagnostic dimensions, the objects differed with respect to their degree of visual angle. Large objects were presented within $\sim 4^{\circ}$ by $\sim 3^{\circ}$ visual angle and small objects within $\sim 2^{\circ}$ by $\sim 1.5^{\circ}$ visual angle. The four objects were situated $\sim 4^{\circ}$ apart in a $2 \times 2$ matrix (see Figure 2). The $2 \times 2$ 


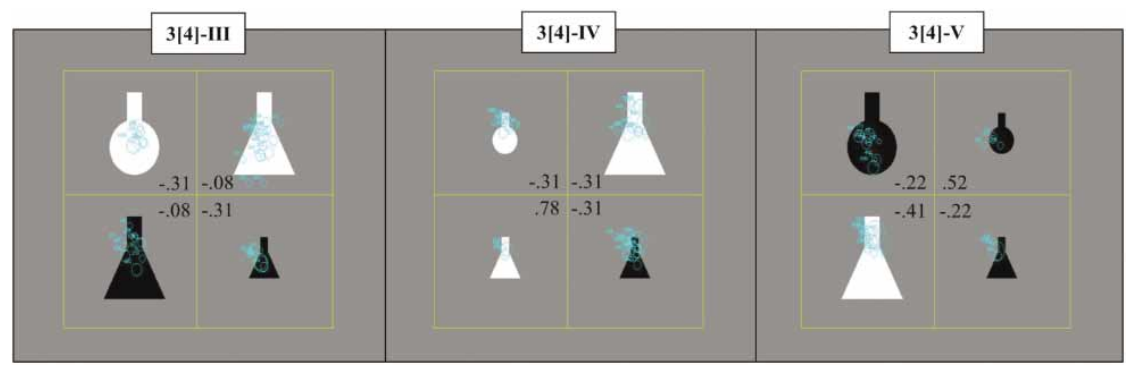

Figure 2. Fixations of a typical participant during the learning phase of a single instance of Types III, IV, and V. Circles are proportional to the duration of fixation with larger circles representing longer fixations. Corresponding information values for each object have been placed within the AOI for that object. Notice how the fixations are biased away from objects with a positive information value towards those objects with negative information values. Note: The rectangles around the objects are the AOIs that were used to count fixations and were not seen by participants during the task. To view this figure in colour, please see the online issue of the Journal.

presentation gives equal emphasis to the four areas of interest (AOIs) and reduces possible biases for particular objects of the set that might be caused by alternative presentations. Square AOIs $\left(\sim 8^{\circ}\right.$ by $8^{\circ}$ visual angle $)$ were drawn around each object and fixations were counted within these AOIs.

\section{Areas of interest}

We defined four square areas of interest (AOIs) drawn around each of the four flasks in the learning phase. All fixations outside of the four AOIs were discarded. Based on the remaining fixations, the dwell time percentage was obtained for each of the four AOIs. The dwell time percentage is the percentage of overall time the participant spent looking inside the given AOI. Dwell time proportions were obtained for each instance of each category structure across all 36 blocks (see Figure 2). Within types, dwell times for structurally equivalent objects were averaged. Table 1 contains the additional dependent variables obtained in the study.

\section{RESULTS AND DISCUSSION}

The ordering of percentage error among the six category structures matched the previously reported pattern (Kruschke, 1992; Love et al., 2004; Nosofsky, Palmeri, \& McKinley, 1994; Vigo, 2011b, 2013b): I (0.01) < II $(0.13)<$ IIII (0.19), IV (0.18), V (0.19)] < VI (0.32). Pairwise $t$-tests showed significantly fewer classification errors for type I than Type II, $t(239)=9.23, p=<.01$; significantly fewer errors for Type II than Types III, IV, and V, least favourable $t(239)=3.25, p<.01$; and significantly fewer errors for Types III, 
TABLE 1

Values of various eyetracking dependent variables during the learning phase of the current experiment

\begin{tabular}{ccc}
\hline & Proportion dwell time & Dwell time (ms) \\
\hline Type I & & \\
1 & 0.236 & 4057 \\
2 & 0.242 & 3841 \\
3 & 0.248 & 2925 \\
4 & 0.234 & 2757 \\
Type II & & \\
1 & 0.246 & 3639 \\
2 & 0.247 & 3682 \\
3 & 0.251 & 3679 \\
4 & 0.246 & 3680 \\
Type III & & \\
1 & 0.343 & 5063 \\
2 & 0.254 & 3759 \\
3 & 0.204 & 3588 \\
4 & 0.187 & 3271 \\
Type IV & & \\
1 & 0.303 & 4824 \\
2 & 0.267 & 4162 \\
3 & 0.210 & 3325 \\
4 & 0.205 & 3236 \\
Type V & & \\
1 & 0.299 & 4624 \\
2 & 0.241 & 3708 \\
3 & 0.230 & 3590 \\
4 & 0.219 & 3402 \\
Type VI & & \\
1 & 0.282 & 3219 \\
2 & 0.240 & 3432 \\
3 & 0.224 & 3877 \\
4 & 0.243 & \\
\hline
\end{tabular}

Proportion dwell time refers to the average proportion of overall fixation time participants spent looking inside a given object's AOI. Dwell time is the average time in milliseconds that participants spent looking inside a given object's AOI.

$\mathrm{IV}$, and V than for Type VI, least favourable $t(239)=6.87, p<.01$. There were no significant differences among Types III, IV, and V.

The gaze of participants reveals a relationship between the structure of the category and the length of fixations for objects within the category (see Figure 3). First, the distribution of gaze across the four objects varies between category structures. Within each structure, the duration of fixation per object varies as a function of the structural context in which the object 

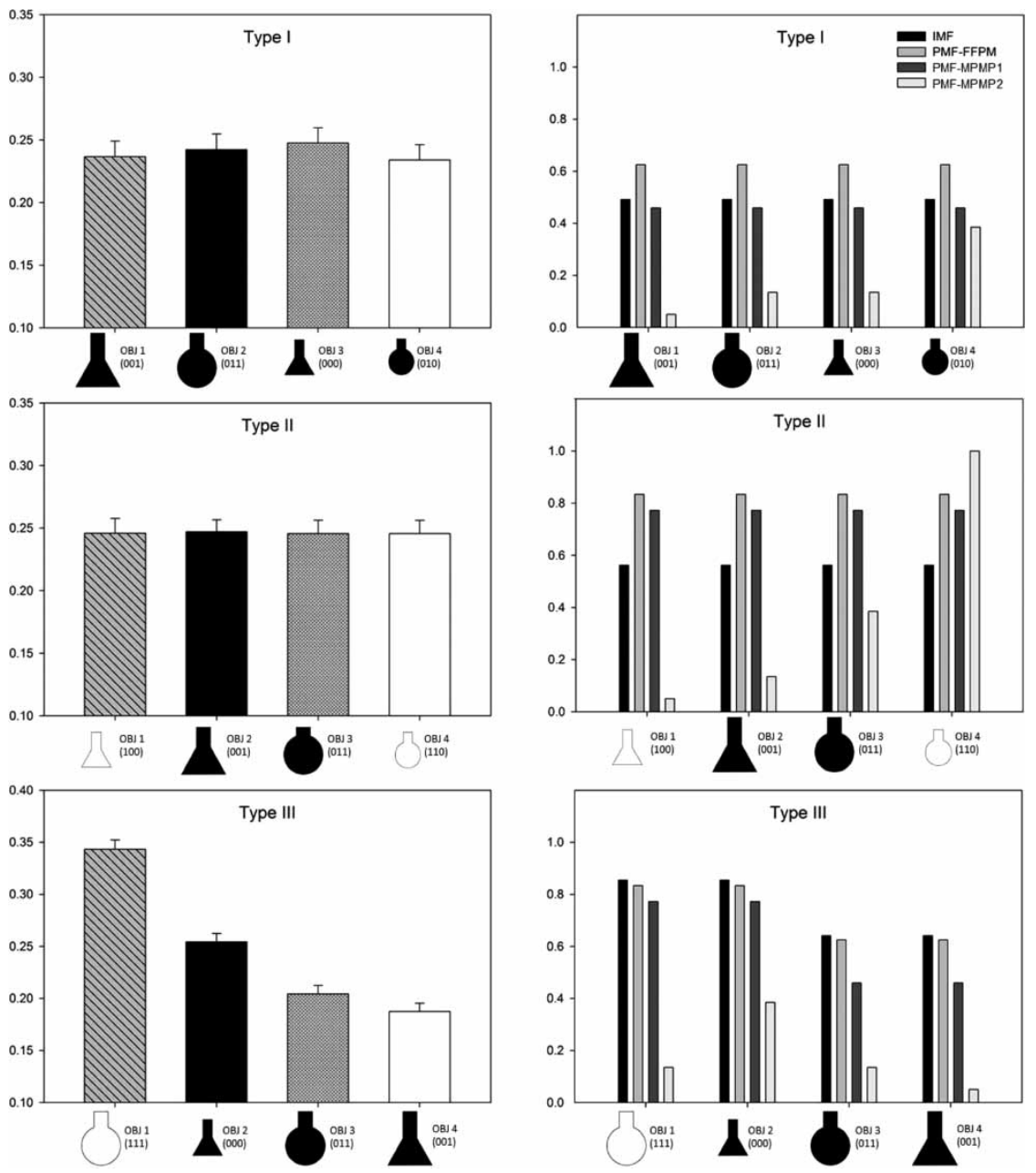

Figure 3. (Left column) Average percentage dwell time while learning three category structures. The horizontal axis displays the informativeness of each object. The vertical axis shows the average percentage dwell time across all 40 subjects. That is, the percentage of time spent looking at each object during the $20 \mathrm{~s}$ learning period. (Right column) The model predictions from the IMF as well as those from the three PMF models (based on the FFPM, Rosch \& Mervis, 1975; MPMP-1, Estes, 1986; MPMP-2, Nosofsky \& Zaki, 2002) are provided. The inverse of the model predictions has been taken (in accordance with the proposed relation) and the result has been standardized in the $0-1$ interval.

belongs. Objects within Types I, II, and VI categories yielded approximately the same amount of fixation time, whereas fixation times on objects within Types III, IV, and V were variable with strong biases for particular objects in the set. The observed fixation durations for objects within a category seem 
highly dependent on its set of origin suggesting a relationship between structural context and visual fixation times. To interpret these findings, we apply a measure of general information known as representational information theory (RIT; Vigo, 2011a) and three well-known measures of prototypicality (Estes, 1986; Nosofsky \& Zaki, 2002; Rosch, 1978), as discussed in the introductory paragraphs.

Overall, we found a general relationship as to the proportion fixation time per object and the amount of information conveyed by each object as measured by RIT. For example, the graphs in Figure 3 show general
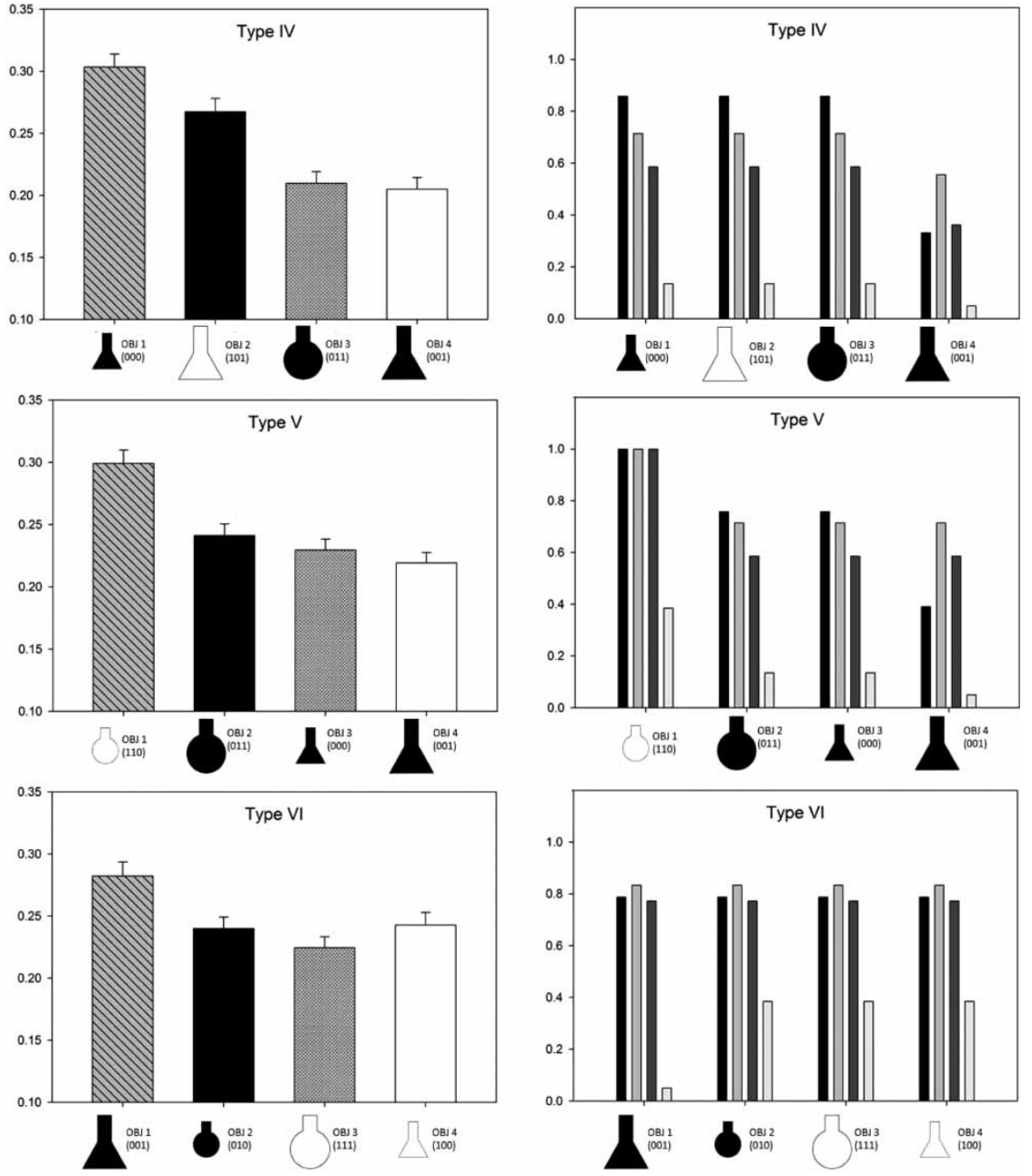

Figure 3. (Continued) 
agreement between the identical information values of object of Types I, II, and VI and their corresponding fixation time per object. In other words, objects within Types I, II, and VI categories yielded approximately the same amount of fixation time as indicated by the least favourable paired samples $t$ test for Type I, $t(239)=0.787, p=.432$, and for Type II, $t(239)=-0.373$, $p=.709(d f=40$ participants multiplied by six trials per type). For Type VI, average differences in fixation time reached significance for only two of the six pairs of paired sample tests. Specifically, there are significant differences between the fixation time of Object 1 and Object 2, $t(237)=2.455, p<.05$, and for Object 1 and Object 3,t $t 237)=3.764, p<.05$. However, note that the nonparametric Mann-Whitney test (see Appendix C) did not yield a significant difference between Objects 1 and 2 of Type VI even though the test corroborated the results of the rest of the pairwise $t$-tests for all six structure types (also, note that results from a multilevel logistic regression analysis shown in Appendix B were consistent with all the $t$-test results reported here). We attribute this slight variation in fixations times between objects of Type VI to the fact that there are only two instances associated with this structure type. That is, only two structurally equivalent instances can be derived given the dimensional assignment of this type. Consequently, whereas structurally equivalent objects were averaged across six instances for Types I-V, the fixation times per object of Type VI were averaged across only two instances. Fixation times for objects within Types III, IV, and V categories indicated a strong negative correlation with their corresponding information values, as illustrated in Figure 3 and Figure 4.

Due to the small number of four points per category type, the qualitative predictions of the model are more compelling. Qualitatively, when learning a category, participants appear to focus on the least informative objects rather than their more informative counterparts. As such, we interpret these results

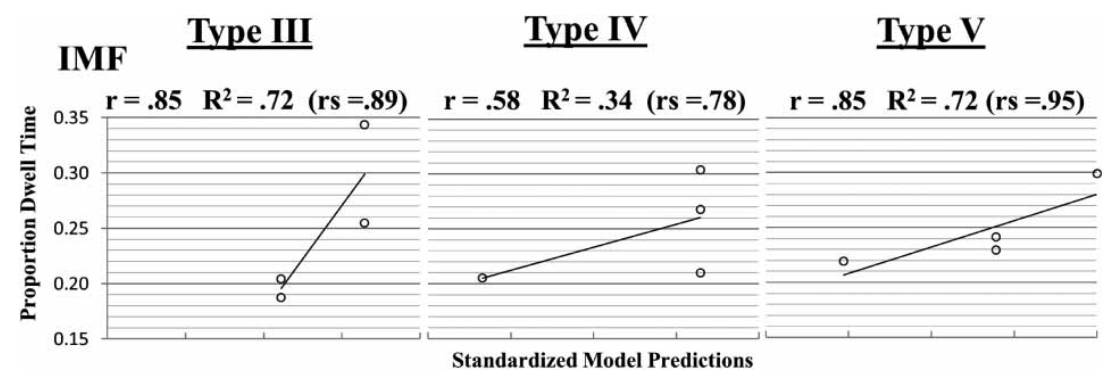

Figure 4. Model fits for the IMF model. The values for the IMF model are obtained by computing the amount of information of each object in each category as specified by representational information theory (Vigo, 2011a, 2013a) and by adding 1 to the results to make any negative values positive. After, the inverse $(1 / x)$ of the values is taken and then standardized on the $[0,1]$ real number interval by using the min/max standardization procedure described in the main text (where the minimum value is set at 0 ). 
by proposing a simple relation; namely, the relative time spent looking at an object during the $20 \mathrm{~s}$ period varies with its level of informativeness as defined in RIT. Specifically, average fixation time for an object during learning was approximately inversely proportional to the amount of information it conveyed about the category containing it. In other words, on average, the less informative an object was about the category containing it (as measured by RIT), the longer it was fixated on relative to the rest of the objects in the category (see Figures 2 and 3). As mentioned, we refer to this relationship as the information model of fixation (IMF). Formally, the information model of fixation (IMF) is defined as follows (where $T_{f}(x \mid \mathrm{C})$ is the proportion dwell time (to the total dwell time for all the objects in C) devoted to object $x$ (of Category $\mathrm{C}$ ) and $\hbar_{s}(x \mid \mathrm{C}$ ) is the amount of representational information conveyed by $x$ about Category C). ${ }^{1}$

$$
T_{f}(x \mid \mathrm{C}) \propto \frac{1}{\hbar_{s}(x \mid \mathrm{C})}
$$

The IMF fits the data accurately as determined by the Spearman's rho test, a test that determines the correlation between the model predictions and the data at an ordinal level: for example, Type III, $r \mathrm{~s}=.89$; Type IV, $r \mathrm{~s}=.78$; Type $\mathrm{V}, r \mathrm{~s}=.95$. Using this test we see that the IMF outperforms the other models considered (see Tables 2 and 3); in particular, the PMF-MPMP2 (i.e., the prototype model of fixation that uses the multiplicative prototype model's notion of prototypicality 2) does not perform as well with respect to category structures of type I, II, III, and VI. Likewise, the PMF-MPMP1 (i.e., the prototype model of fixation that uses the multiplicative prototype model's notion of prototypicality 1) and the PMF-FFPM (i.e., the prototype model of fixation that uses the feature frequency prototype model) do not perform as well with respect to type $\mathrm{V}$ category structures. In addition, on average, the IMF accounts for most of the variance in the data: type III; $R^{2}=.72, r=.85$, type IV; $R^{2}=.34, r=.58$, type $\mathrm{V} ; R^{2}=.72, r=.85$ (see Figure 4). In this respect, the IMF outperforms the PMF-MPMP2 and performs, on average, at about the same level as the PMF-FFPM and the PMF-MPMP1 models (again, see Table 2 and 3 for the model predictions). However, as mentioned, the small number of objects (four) per category

\footnotetext{
${ }^{1}$ By including a scaling parameter $b$ (where $b$ is a positive real number) for scaling information units to units of time, the IMF is defined as shown below where $T_{F}(x \mid \mathrm{C})$ may then be regarded as the fixation time devoted to object $x$. Please note that in this paper, we only discuss and fit the data with the parameter-free version of the IMF introduced earlier and not with this version.
}

$$
T_{f}(x \mid \mathrm{C}) \propto \frac{1}{b \cdot \hbar_{s}(x \mid \mathrm{C})}
$$


TABLE 2

Model predictions for the IMF and PMF models per object within each of the six categories of objects associated with 3[4] family of category structures

\begin{tabular}{|c|c|c|c|c|c|}
\hline Type & $\begin{array}{c}I M F \\
(\text { Vigo, 2011a) }\end{array}$ & $\begin{array}{l}P M F-F F P M \\
(\text { Rosch, 1978) }\end{array}$ & $\begin{array}{c}P M F-M P M P-1 \\
(\text { Estes, 1986, 1994) }\end{array}$ & $\begin{array}{c}P M F-M P M P-2 \\
\text { (Nosofsky \& Zaki, 2002) }\end{array}$ & $\begin{array}{l}\text { Proportion dwell time } \\
\text { (Vigo et al.) }\end{array}$ \\
\hline $3[4]-1$ & {$[.492, .492, .492, .492]$} & {$[.625, .625, .625, .625]$} & {$[.459, .459, .459, .459]$} & {$[.05, .135, .135, .385]$} & {$[.236, .242, .248, .234]$} \\
\hline $3[4]-3$ & {$[.855, .855, .641, .641]$} & {$[.833, .833, .625, .625]$} & {$[.773, .773, .460, .460]$} & {$[.135, .385, .135, .05]$} & {$[.343, .254, .204, .187]$} \\
\hline $3[4]-4$ & {$[.855, .855, .855, .331]$} & {$[.714, .714, .714, .555]$} & {$[.586, .586, .586, .362]$} & {$[.135, .135, .135, .05]$} & {$[.303, .267, .210, .205]$} \\
\hline $3[4]-5$ & {$[1, .756, .756, .388]$} & {$[1, .714, .714, .714]$} & {$[1, .586, .586, .586]$} & {$[.385, .135, .135, .05]$} & {$[.299, .241, .230, .219]$} \\
\hline $3[4]-6$ & {$[.787, .787, .787, .787]$} & {$[.833, .833, .833, .833]$} & {$[.773, .773, .773, .773]$} & {$[.05, .385, .385, .385]$} & {$[.282, .240, .224, .243]$} \\
\hline
\end{tabular}

Values per object should be interpreted as the inverse of representational information values (in the case of the IMF model) and the inverses of the degrees of prototypicality (in the case of the PMF models). The predictions have been standardized in the $[0,1]$ real number interval as specified earlier. Average proportion dwell time from the current experiment has been included in the last column. 
TABLE 3

Correlations between model predictions and proportion dwell time per object

\begin{tabular}{lllll}
\hline & $I M F$ & $P M F-F F P M$ & $P M F-M P M P-1$ & $P M F-M P M P-2$ \\
\hline Type III & $.85(.72)$ & $.85(.72)$ & $.85(.72)$ & $.23(.05)$ \\
& $\mathbf{. 8 9}$ & $\mathbf{. 8 9}$ & $\mathbf{. 8 9}$ & $\mathbf{. 6 3}$ \\
Type IV & $.58(.34)$ & $.58(.34)$ & $.58(.34)$ & $.58(.34)$ \\
& $\mathbf{. 7 8}$ & $\mathbf{. 7 8}$ & $\mathbf{7 8}$ &. $\mathbf{7 8}$ \\
Type V & $.85(.72)$ & $.97(.94)$ & $.97(.94)$ & $.99(.98)$ \\
& $\mathbf{. 9 5}$ & $\mathbf{. 7 8}$ & $\mathbf{. 7 8}$ & .95 \\
\hline
\end{tabular}

Reported in this table are Pearson's $r$ correlations, $R^{2}$ values (in parentheses), and Spearman's rho values (in bold).

renders the $R^{2}$ statistic less telling and useful. Thus, in general, considering the qualitative predictions of Table 2 along with Spearman's ordinal correlations, the IMF is the best performing model.

We also examined the dynamics of eye fixation by taking the moving averages of the fixation times across trials per structure type (see Appendix A for these results). As shown in the graphs of Appendix A, these averages converged and seem to have reached a steady-state in agreement with the model predictions. This is interesting because it seems to indicate that after exposure to only a few interspersed structure instances, humans reach asymptotic fixation behaviour. In other words, humans seem to be able to extract contextual or structural relationships between the objects of the categories efficiently.

From this connection, it would seem that, because informative objects are learned quickly, participants spend more time fixating on less informative and less representative objects. Additionally, both the direction and magnitude of the information values seem to affect fixations in the way that gaze is directed away from objects with positive information values toward those with negative information values. And as hypothesized, the structure of the category seems to influence fixation times in a predictable manner.

Using the data obtained from the current study, the graphs in the right column of Figure 3 illustrate the PMF predictions for each of the measures of prototypicality. The objects that receive the least amount of fixation time during learning are those that are most prototypical of the category (see Figure 5 for model plots). An important distinction between the IMF and the three versions of the PMF is that information as described by RIT in the IMF is based solely on the objects in the set, whereas under the PMF account a hypothesized representation (i.e., the prototype) is formulated by the human conceptual system and exists outside the set of objects. Table 2 shows the values obtained for RIT and the three prototype accounts just described. Overall, the IMF appears to predict fixation times, at a qualitative 


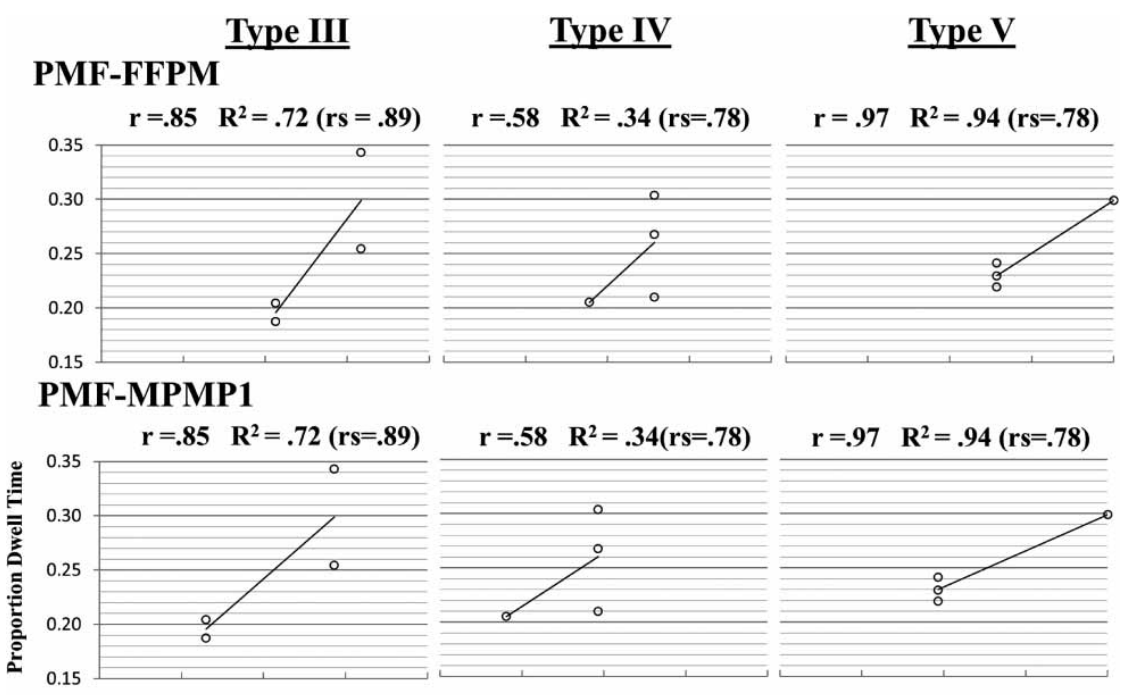

PMF-MPMP2

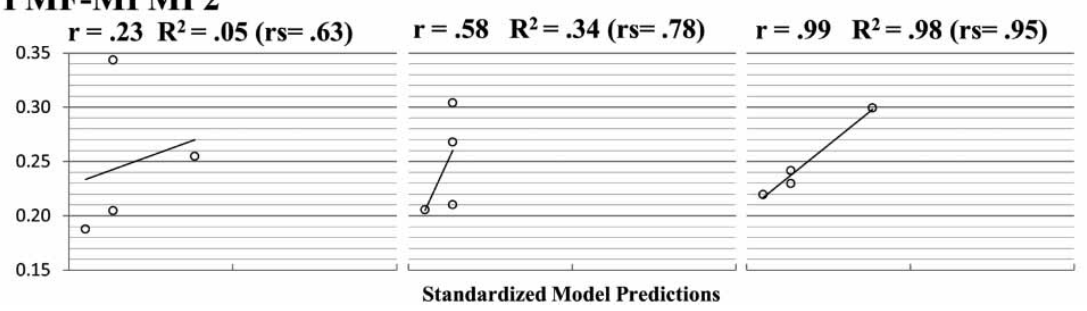

Figure 5. Model fits for PMF models, including FFPM, MPMP-1, and MPMP-2. The values for the PMF models are obtained by computing the degree of prototypicality of each object in each category as specified by the given model/measure. After, the inverse $(1 / x)$ of the values is taken and then standardized on the $[0,1]$ real number interval by using the min/max standardization procedure described in the main text (where the minimum value is set at 0 ).

and ordinal level, more accurately than the three versions of the PMF. Moreover, the PMF-MPMP-2 does not perform as well as the IMF with respect to four category structures (Types I, II, III, and VI), more than the PMF models. Further analysis using the proposed framework showed that a fourth measure of degree of prototypicality proposed by Smith, Murray, and Minda's (1997) yields the exact same fits to our data as does the MPMP-1. For this reason, the predictions of this model are not shown in our data plots. Finally, the model performance results summarized in this section are consistent with a recent finding by Vigo and Baswaraj (2013). These researchers showed that RIT outperforms prototype theories when participants are asked to give explicit magnitude judgements of informativeness. 
The values for the PMF models are obtained by first calculating the degree of prototypicality for an object as specified by the given model (see Vigo \& Baswaraj, 2013, for these values). Once the values are obtained, the inverse (1/ $x$ ) is taken in accordance with the inverse relation found in our experiment. Next, in order to standardize the values on the $[0,1]$ real number interval, we use a min/max procedure where each inverse value $z$ is recalculated as $(z-\min ) /$ (max-min), where $\min$ is the minimal value (set to zero), and $\max$ is the maximum value across all six category structure types. Due to the fact that RIT yields negative representational information values for some objects, the additional standardization step of adding one to the original information values is taken before taking the inverse and standardizing.

Please note that although some researchers may formulate interpretations in terms of other constructs, such as utility (Nelson \& Cotrell, 2007), entropy limit minimization (Najemnik \& Geisler, 2009), and value-based decisions (Krajbich \& Rangel, 2011), we feel that such efforts are driven by significantly different goals. Our aim is to try to account for the observed patterns using a measure that was meant to characterize information in general (i.e., not in a domain or phenomena-specific fashion) and that is highly sensitive to structural context. Accordingly, the application of RIT in this paper is to provide a direct conceptual interpretation of our experimental data linking object gaze to the amount and quality of the information (i.e., information in an abstract and general sense) that an object conveys. A similar direct link was established by Vigo and Baswaraj (2013) with respect to informativeness judgements but not for eye movements.

\section{CONCLUSION AND FUTURE DIRECTIONS}

We used eye tracking and a novel empirical classification paradigm to investigate the link between average fixation times on the objects of a category and the amount of information conveyed by the objects about the category. The data from our experiment suggests an inverse relation between average fixation time on an object and its degree of informativeness. The good fit between the information model of fixation (IMF) and the data was encouraging. This is particularly true in light of a recent behavioural finding by Vigo and Baswaraj (2013) regarding informativeness judgements. In their investigation, these researchers found a near perfect link between informativeness magnitude judgements and representational information using categorical stimuli also consisting of three dimensions and four objects.

Although the IMF has an advantage over the prototype models of fixation, it did not fit the data as accurately as we had hoped, in particular for Type III category structures. From this we conclude that the dwell time patterns observed in our data should be interpreted only as approximations 
of the performance cost involved in the mental process of information assessment. Automatic motor propensities may "absorb" a significant portion of the variance in any eye-tracking data that purports to establish a connection between eye gaze and a higher order cognitive facility (Rolfs, 2009). In short, although eye fixations will reveal much of the temporal cost involved in higher order informativeness assessments, it will not reveal all. A refinement of the proposed inverse relation model that would account for this additional variance can be achieved by simply adding such response bias parameters. In addition, one should consider that the presentation intervals may have influenced the results of the current experiment. For instance, if the presentation time during the learning phase is longer than the time needed to learn the category, additional eye movements may not reflect the processes involved in learning the category. Likewise, if the presentation is shorter than the time needed to learn the category it may cause eye movements that could have been related to category relevance to be excluded from the data analysis.

A limitation of the current research is that only dwell times are considered in the analysis of eye movements. Yet, the pattern of eye movements (i.e., the order in which the objects are looked at) can reveal much about how participants go about gathering information about the objects. We regard this type of sequential analysis as an important direction to pursue in future research. Notwithstanding its current limitations, the proposed study extends to fields with an increasing concern for the factors that influence fixation duration during decision making. An expanding literature in this regard is evident in consumer research (Ozanne, Brucks, \& Grewal, 1992; Reutskaja, Nagel, Camerer, \& Rangel, 2011), human-computer interface (Cheng \& Liu, 2011; Pan et al., 2004) as well as the combination of the two, i.e., online marketing (Djamasbi, Siegel, Skorinko, \& Tullis, 2011; Dreze \& Hussherr, 2003). As far as we know, such a bias for less typical objects in a display has only been shown by the novelty and familiarity effects that occur during infant preference paradigms (Furrer \& Younger, 2005; Oakes \& Riber, 2005; Quinn, 2004; Quinn \& Bhatt, 1998). The information-fixation link presented here is applicable to any circumstance where manipulation of gaze might be useful. For example, we can see how this relationship has broad implications for marketing with respect to product placement.

More specifically, using these findings, a seller can manipulate the amount of time that an individual fixates on a particular item (among a set of items) when the ultimate goal of the consumer is to gather relative information about each item. The empirical results of the experiment reported here can be used to serve this goal. Take, for example, four toy model cars (A, B, C, and D) that are on display in a store. Let us suppose that the dimensions of interest to most collectors are colour, size, and general shape (e.g., curvy vs. flat) and that the four cars could be chosen so that the relationship between their dimensional values conform to any one of the six 3[4] structures. 
Furthermore, suppose that the goal of the store is for collectors to, until they are approached by a salesman, spend relatively more time looking at toy model car A. The question is: Which context, or equivalently, which of the six structures should be reflected by the set of four toy model cars in order to achieve this goal? According to the IMF, the right structure to choose is the one where toy model car A is the least informative among the four.

Furthermore, future studies should aim to specify the difference between the two levels of information processing (featural vs. whole level processing) during category learning. The difference between the feature level information processing presented by Rehder and Hoffman (2005) and the whole object information processing presented here should be further investigated in order to better understand the role of gaze while learning category membership. The level of processing used by participants is likely a function of the differing tasks used in each of the studies. Recently, researchers presented an account of feature processing in categorization using both spatially separated dimensions as well as features that are part of the same object (see Little, Nosofsky, Donkin, \& Denton, 2012). Spatially separated dimensions were found to be processed in a serial fashion regardless of whether or not they were part of the same object. Notably, Little, Nosofsky, and Denton (2011) used spatially separated but overlapped dimensions.

Therefore, further investigation is needed to fully understand: (1) the conditions under which different levels of processing are utilized, (2) whether or not whole objects are the primary unit of processing, and (3) whether features of those objects are processed simultaneously. Indeed, it could be that eye movements at the featural level may predict eye movements at the whole level. In this way, low-level processing may be able to predict fixations at higher levels of processing, but the appropriate study would have to be done in order to investigate this relationship.

Moreover, we pointed at several theoretical advantages that representational information theory (RIT) holds over the three tested models of prototypicality. However, we did not compare the IMF to categorization models based on prototypes. In other words, we tested models/measures of prototypicality at the core of these models but not the models themselves. The reason is that these models yield the probability of classifying an item correctly based on its similarity to a prototype (as defined by the theory). But our interest and focus was on computing the most prototypical item of a set of objects as a way of extracting its most representative element - a very different construct, indeed, and one that compares meaningfully to one of the core aspects of representational information. Notwithstanding, it might be worthwhile to explore the connection between classification probabilities of objects as predicted by these models and fixation times. This prospect opens the way to other kinds of classification models. For example, the "rule plus exception model", or RULEX (Nosofsky, Palmeri, \& McKinley, 1994) 
predicts that objects that do not fit a category rule take longer to process. This suggests that such objects necessitate longer fixations. Palmeri and Nosofsky (1995) show that these category exceptions are categorized more slowly than items which adhere to the category rule. The varying fixations for objects in category Types III, IV, and V could therefore be due to the several rule-plus-exception (RULEX) strategies indicated by this account. The relationship between the rule-plus exception model and the measure of representational information should be explored and the extent to which RULEX accounts for fixation durations is a future goal of the current research.

Additionally, researchers have sought to describe this process of information acquisition during category learning where information search has been described using a Bayesian framework (Nelson \& Cotrell, 2007) and utility functions can be used to describe the expected information value of particular questions or queries. Beliefs, in this case, are described in terms of subjective probabilities and Bayes' theorem can be used to update beliefs as new information is provided (see Nelson, 2005). Alternatively, information acquisition has been described by the process of maximizing probability gain (Nelson, McKenzie, Cottrell, \& Sejnowski, 2010). Maximizing probability gain is similar to the notion of error reduction that is traditionally incorporated in learning models (see Kruschke, 2003).

In conclusion, the way that complex eye movements hold external clues to the inner works of higher order information processing when analysed using RIT and GRIT (its generalization to continuous domains), demonstrates the potential of the theory's central measure in increasing our understanding of the link between physiological and mental processes. We believe that several disparate lines of research may be unified under this general measure and hope that the robustness of the mathematical link between representational information and gaze described by the IMF is tested further as it extends to other domains.

\section{REFERENCES}

Bourne, L. E. (1966). Human conceptual behavior. Boston, MA: Allyn \& Bacon.

Cheng, S., \& Liu, Y. (2012). Eye-tracking based adaptive user interface: implicit humancomputer interaction for preference indication. Journal on Multimodal User Interfaces, 5, $77-84$.

Djamasbi, S., Siegel, M., Skorinko, J., \& Tullis, T. (2011). Online Viewing and Aesthetic Preferences of Generation Y and the Baby Boom Generation: Testing User Web Site Experience Through Eye Tracking. International Journal of Electronic Commerce, 15, 121-158.

Dreze, X., \& Hussherr, F. X. (2003). Internet advertising: Is anybody watching? Journal of interactive marketing, 17, 8-23.

Estes, W. K. (1986). Array models for category learning. Cognitive psychology, 18, 500-549.

Estes, W. K. (1994). Classification and cognition (Oxford Psychology Series, No. 22). Oxford: Oxford University Press. 
Furrer, S. D., \& Younger, B. A. (2005). Beyond the distributional input? A developmental investigation of asymmetry in infants' categorization of cats and dogs. Developmental Science, 8, 544-550. doi:10.1111/j.1467-7687.2005.00446.x

Garner, W. R. (1974). The processing of information and structure. New York, NY: Wiley.

Hayhoe, M., \& Ballard, D. (2005). Eye movements in natural behavior. Trends in Cognitive Sciences, 9, 188-194. doi:10.1016/j.tics.2005.02.009

Henderson, J. M., \& Hollingworth, A. (1999). High-level scene perception. Annual Review of Psychology, 50, 243-271. doi:10.1146/annurev.psych.50.1.243

Kowler, E. (1991). The role of visual and cognitive processes in the control of eye movement. In E. Kowler (Ed.), Eye Movements and Their Role in Visual and Cognitive Processes (pp. 1-70). Amsterdam: Elsevier.

Krajbich, I., \& Rangel, A. (2011). A multi-alternative drift diffusion model predicts the relationship between visual fixations and choice in value-based decisions. Proceedings of the National Academy of Sciences, 108, 13852-13857. doi:10.1073/pnas.1101328108

Kruschke, J. K. (1992). ALCOVE: An exemplar-based connectionist model of category learning. Psychological Review, 99, 22-44. doi:10.1037/0033-295X.99.1.22

Kruschke, J. K. (2003). Attention in learning. Current Directions in Psychological Science, 12, 171-175. doi:10.1111/1467-8721.01254

Little, D. R., Nosofsky, R. M., \& Denton, S. E. (2011). Response-time tests of logical-rule models of categorization. Journal of Experimental Psychology: Learning, Memory, and Cognition, 37, 1-27. doi:10.1037/a0021330

Little, D. R., Nosofsky, R. M., Donkin, C., \& Denton, S. E. (2012). Logical Rules and the Classification of Integral-Dimension Stimuli. Journal of experimental psychology. Learning, memory, and cognition, 39, 801-820.

Liversedge, S. P., \& Findlay, J. M. (2000). Saccadic eye movements and cognition. Trends in Cognitive Sciences, 4, 6-14. doi:10.1016/S1364-6613(99)01418-7

Love, B. C., Medin, D. L., \& Gureckis, T. M. (2004). SUSTAIN: A network model of category learning. Psychological Review, 111, 309-332. doi:10.1037/0033-295X.111.2.309

Martinez-Conde, S., Macknik, S. L., \& Hubel, D. H. (2004). The role of fixational eye movements in visual perception. Nature Reviews Neuroscience, 5, 229-240. doi:10.1038/nrn1348

Medin, D. L., \& Schaffer, M. M. (1978). Context theory of classification learning. Psychological Review, 85, 207-238. doi:10.1037/0033-295X.85.3.207

Mele, M. L., \& Federici, S. (2012). Gaze and eye-tracking solutions for psychological research. Cognitive processing, 13, 261-265.

Najemnik, J., \& Geisler, W. S. (2009). Simple summation rule for optimal fixation selection in visual search. Vision Research, 49, 1286-1294. doi:10.1016/j.visres.2008.12.005

Nelson, J. D. (2005). Finding useful questions: On Bayesian diagnosticity, probability, impact, and information gain. Psychological Review, 112, 979-999. doi:10.1037/0033-295X.112.4.979

Nelson, J. D. \& Cottrell, G. W. (2007). A probabilistic model of eye movements in concept formation. Neurocomputing, 70, 2256-2272. doi:10.1016/j.neucom.2006.02.026

Nelson, J. D., McKenzie, C. R. M. Cottrell, G. W., \& Sejnowski, T. J. (2010). Experience matters: Information acquisition optimizes probability gain. Psychological Science, 21, 960 969. doi:10.1177/0956797610372637

Nosofsky, R. M., Gluck, M. A., Palmeri, T. J., Mckinley, S. C., \& Glauthier, S. C. (1994). Comparing models of rule-based classification learning: A replication and extension of Shepard, Hovland, and Jenkins (1961). Memory and Cognition, 24, 352-369.

Nosofsky, R. M. Palmeri, T. J., \& McKinley, S. C. (1994). Rule-plus-exception model of classification learning. Psychological Review, 101, 53-79. doi:10.1037/0033-295X.101.1.53

Nosofsky, R. M., \& Zaki, S. R. (2002). Exemplar and prototype models revisited: Response strategies, selective attention, and stimulus generalization. Journal of Experimental Psychology: Learning, Memory, and Cognition, 28, 924-940. doi:10.1037/0278-7393.28.5.924 
Oakes, L. M., \& Ribar, R. J. (2005). A comparison of infants' categorization in paired and successive presentation familiarization tasks. Infancy, 7, 85-98. doi:10.1207/s15327078in0701_7

Ozanne, J. L. Brucks, M., \& Grewal, D. (1992). A study of information search behavior during the categorization of new products. Journal of Consumer Research, 18, 452-463. doi:10.1086/ 209273

Palmeri, T. J., \& Nosofsky, R. M. (1995). Recognition memory for exceptions to the category rule. Journal of Experimental Psychology: Learning, Memory, and Cognition, 21, 548-568. doi:10.1037/0278-7393.21.3.548

Pan, B., Hembrooke, H. A., Gay, G. K., Granka, L. A., Feusner, M. K., \& Newman, J. K. (2004, March). The determinants of web page viewing behavior: an eye-tracking study. In Proceedings of the 2004 symposium on Eye tracking research \& applications (pp. 147-154). San Antonio, TX. ACM.

Quinn, P. C. (2004). Development of subordinate-level categorization in 3-to 7-month-old infants. Child Development, 75, 886-899. doi:10.1111/j.1467-8624.2004.00712.x

Quinn, P. C., \& Bhatt, R. S. (1998). Visual pop-out in young infants: Convergent evidence and an extension. Infant Behavior and Development, 21, 273-288. doi:10.1016/S01636383(98)90006-6

Rayner, K. (1998). Eye movements in reading and information processing: 20 years of research. Psychological Bulletin, 124, 372-422. doi:10.1037/0033-2909.124.3.372

Rayner, K., Smith, T. J., Malcolm, G. L., \& Henderson, J. M. (2009). Eye movements and visual encoding during scene perception. Psychological Science, 20, 6-10. doi:10.1111/j.14679280.2008.02243.x

Rehder, B., \& Hoffman, A. B. (2005). Eyetracking and selective attention in category learning. Cognitive Psychology, 51, 1-41. doi:10.1016/j.cogpsych.2004.11.001

Reutskaja, E., Nagel, R., Camerer, C. F., \& Rangel, A. (2011). Search dynamics in consumer choice under time pressure: An eye-tracking study. The American Economic Review, 101, 900-926.

Rolfs, M. (2009). Microsaccades: Small steps on a long way. Vision Research, 49, 2415-2441. doi:10.1016/j.visres.2009.08.010

Rosch, E. (1978). Principles of categorization. In E. Rosch \& B. B. Lloyd (Eds.), Cognition and categorization (pp. 27-48). Hillsdale, NJ: Lawrence Erlbaum Associates, Inc. Reprinted in E. Margolis \& S. Laurence (Eds.). (1999), Concepts: Core readings (pp. 189-206). Cambridge, MA: MIT Press.

Rosch, E., \& Mervis, C. B. (1975). Family resemblances: Studies in the internal structures of categories. Cognitive Psychology, 7, 573-605. doi:10.1016/0010-0285(75)90024-9

Shepard, R. N., Hovland, C. L., \& Jenkins, H. M. (1961). Learning and memorization of classifications. Psychological Monographs: General and Applied, 75, 1-42. doi:10.1037/ h0093825

Shepard, R. N. (1974). Representation of structure in similarity data: Problems and prospects. Psychometrika, 39, 373-421.

Shepard, R. N. (1987). Toward a universal law of generalization for psychological science. Science, 237, 1317-1323.

Smith, J. D., Murray, M. J., \& Minda, J. P. (1997). Straight talk about linear separability. Journal of Experimental Psychology: Learning, Memory, and Cognition, 23, 659-680. doi:10.1037/ 0278-7393.23.3.659

Vigo, R. (2009a). Categorical invariance and structural complexity in human concept learning. Journal of Mathematical Psychology, 53, 203-221.

Vigo, R. (2009b). Modal similarity. Journal of Experimental and Theoretical Artificial Intelligence, 21, 181-196.

Vigo, R., Allen, C. (2009c). How to reason without words: inference as categorization. Cognitive Processing, 10, 77-88. 


\section{4}

Vigo, R. (2011a). Representational information: A new general notion and measure of information. Information Sciences, 181, 4847-4859.

Vigo, R. (2011). Towards a Law of Invariance in Human Conceptual Behavior. In Proceedings of the 33rd Annual Meeting of the Cognitive Science Society (pp. 2580-2585). Cognitive Science Society.

Vigo, R. (2013a). Meaning over uncertainty in Generalized Representational Information Theory (GRIT): A structure sensitive theory of information. Information, 4, 1-30.

Vigo, R. (2013b). The GIST of concepts. Cognition. doi:10.1016/j.cognition.2013.05.008

Vigo, R., \& Basawaraj, B. (2013). Will the most informative object stand? Determining the impact of structural context on informativeness judgements. Journal of Cognitive Psychology, 1-19. (in press)

Viviani, P. (1990). Eye movements in visual search: cognitive, perceptual and motor control aspects. In E. Kowler (Ed.), Eye Movements and Their Role in Visual and Cognitive Processes (pp. 253-393). Amsterdam: Elsevier.

Yarbus, A. L. (1967). Eye-movements and vision. New York, NY: Plenum Press.

Manuscript received August 2012 Revised manuscript received April 2013

First published online May 2013 


\section{TECHNICAL APPENDIX}

The following explanation is similar to that presented in the technical appendix of Vigo and Basawaraj (2013). A well-defined category is a set containing objects that are defined in terms of a preset finite number of dimensions and their values. In Representational Information Theory (RIT; Vigo, 2011a), the amount and quality of subjective information $\hbar_{S}$ conveyed by a subset $\mathrm{R}$ of a well-defined category $\widehat{F}$ is defined as the proportional or percent change (i. e., the percent change divided by 100) in the perceived (or subjective) structural complexity $\psi$ of $\widehat{F}$ whenever $\mathrm{R}$ is subtracted from $\widehat{F}$. This is expressed by the following equation where $R \subseteq \widehat{F}$ or equivalently, $R \in \wp(\widehat{F}), \widehat{G}=\widehat{F}-R,|\widehat{G}|$ and $|\widehat{F}|$ are the number of elements in $\widehat{G}$ and $\widehat{F}$ respectively, and $\phi$ is the degree of categorical invariance of a well-defined category of objects (see Vigo, 2009a, 2011a, 2011b, 2013b for a detailed discussion of structural complexity and categorical invariance).

$$
\hbar_{S}(R \mid \widehat{F})=\frac{\psi(\widehat{G})-\psi(\widehat{F})}{\psi(\widehat{F})}=\frac{|\widehat{G}| e^{-\Phi(G(\overrightarrow{\mathrm{x}}))}-\left(|\widehat{F}| e^{-\Phi(F(\overrightarrow{\mathrm{x}}))}\right)}{\left(|\widehat{F}| e^{-\Phi(F(\overrightarrow{\mathrm{x}}))}\right)}
$$

The degree of categorical invariance $\Phi$ of $\widehat{F}$ is defined with the following metric:

$$
\Phi(F)=\left[\sum_{i=1}^{D}\left[\left\|\frac{\widehat{\partial} F\left(x_{1}, \cdots, x_{D}\right)}{\widehat{\partial} x_{i}}\right\|\right]^{2}\right]^{1 / 2}
$$

where

$$
\left\|\frac{\hat{\partial} F\left(x_{1}, \cdots, x_{D}\right)}{\hat{\partial} x_{i}}\right\|=1-\left[\frac{1}{p} \sum_{\vec{x}_{j} \in \hat{F}}\left|\frac{\hat{\partial} F\left(\vec{x}_{j}\right)}{\hat{\partial} x_{i}}\right|\right]
$$

In the above definition, $\vec{x}$ stands for an object defined by $D$ dimensional values $\left(x_{1}, \ldots, x_{D}\right)$. The general summation symbol represents the sum of the partial derivatives evaluated at each object $\vec{x}_{j}$ from the Boolean category $\widehat{F}$ (this is the category defined by the concept function $F$ ). The partial derivative transforms each object $\vec{x}_{j}$ in respect to its $i$-th dimension and evaluates to 0 if, after the transformation, the object is still in $\widehat{F}$ (it evaluates to 1 otherwise). Thus, to compute the proportion of objects that remain in $\widehat{F}$ after changing the value of their $i$-th dimension, we need to divide the sum of the partial derivatives evaluated at each object $\vec{x}_{j}$ by $p$ (the number of 
objects in $\widehat{F}$ ) and subtract the result from 1 . The absolute value symbol is placed around the partial derivative to avoid a value of negative 1. For a detailed explanation of these measures and concepts see Vigo (2009a, 2011a, 2011a). Although the measures defined here work only on categories defined over binary dimensions, the theory has been generalized to continuous dimensions in Generalized Representational Information Theory (GRIT; Vigo, 2013a).

\section{Example:}

With RIT we may calculate the amount of subjective representational information associated with the objects that constitute each type of the 3[4] category structure. Take, for instance, the categorical stimulus shown here:

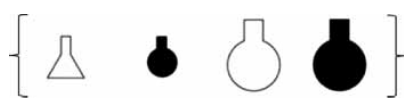

If we wish to compute the informativeness of the first object in this set (i.e., the small, white, triangular flask), we first compute the subjective structural complexity (using the equations above) of the category as a whole $\psi(\widehat{F})$ as well as the subjective complexity of the subset composed of the three remaining objects after the first object is removed $\psi(\widetilde{G})$. The subjective complexity of the two sets $\widehat{F}$ and $\widehat{G}$ may now be computed as calculated using the equation defined earlier:

$$
\begin{aligned}
\psi(\widehat{F}) & =|\widehat{F}| e^{-\Phi(F(\vec{x}))}=4 e^{-.71}=1.97 \\
\psi(\widehat{G}) & =|\widehat{G}| e^{-\Phi(G(\vec{x}))}=3 e^{-.94}=1.17
\end{aligned}
$$

The values of $\psi(\widehat{F})$ and $\psi(\widehat{G})$ are then used to measure the amount of representational information conveyed by the particular object, i.e., the rate of change in the subjective structural complexity when the particular object is removed from the set as follows (please note that the images here are meant for friendly illustration only and that, formally, categories and their elements are represented in terms of vectors of dimensional values):

$$
h_{s}(\Delta \mid[\Delta \boldsymbol{\delta}])=\frac{\psi(\widehat{G})-\psi(\widehat{F})}{\psi(\widehat{F})}=\frac{1.17-1.97}{1.97} \approx-.41
$$

Using this procedure we can calculate information values for each element in any dimensionally defined set. If the procedure is continued for the remaining elements of the stimulus in question, we arrive at the following information values, respectively: $-.22,-.22, .52$. This example illustrates 
that the small, white, triangular flask is relatively less informative with respect to its category of origin since its absence results in a $41 \%$ reduction in the perceived complexity (or in the degree of concept learning difficulty) of the category.

\section{APPENDIX A: MOVING AVERAGES}

The line plots in Figure A1 track the average dwell time of structurally equivalent objects within each type across the six trials in the current experiment. The objects (1-4) in these plots are labelled in accordance with Figure 3. Note that by the fifth and sixth trials, the graphs converge in agreement with the models' predictions. 

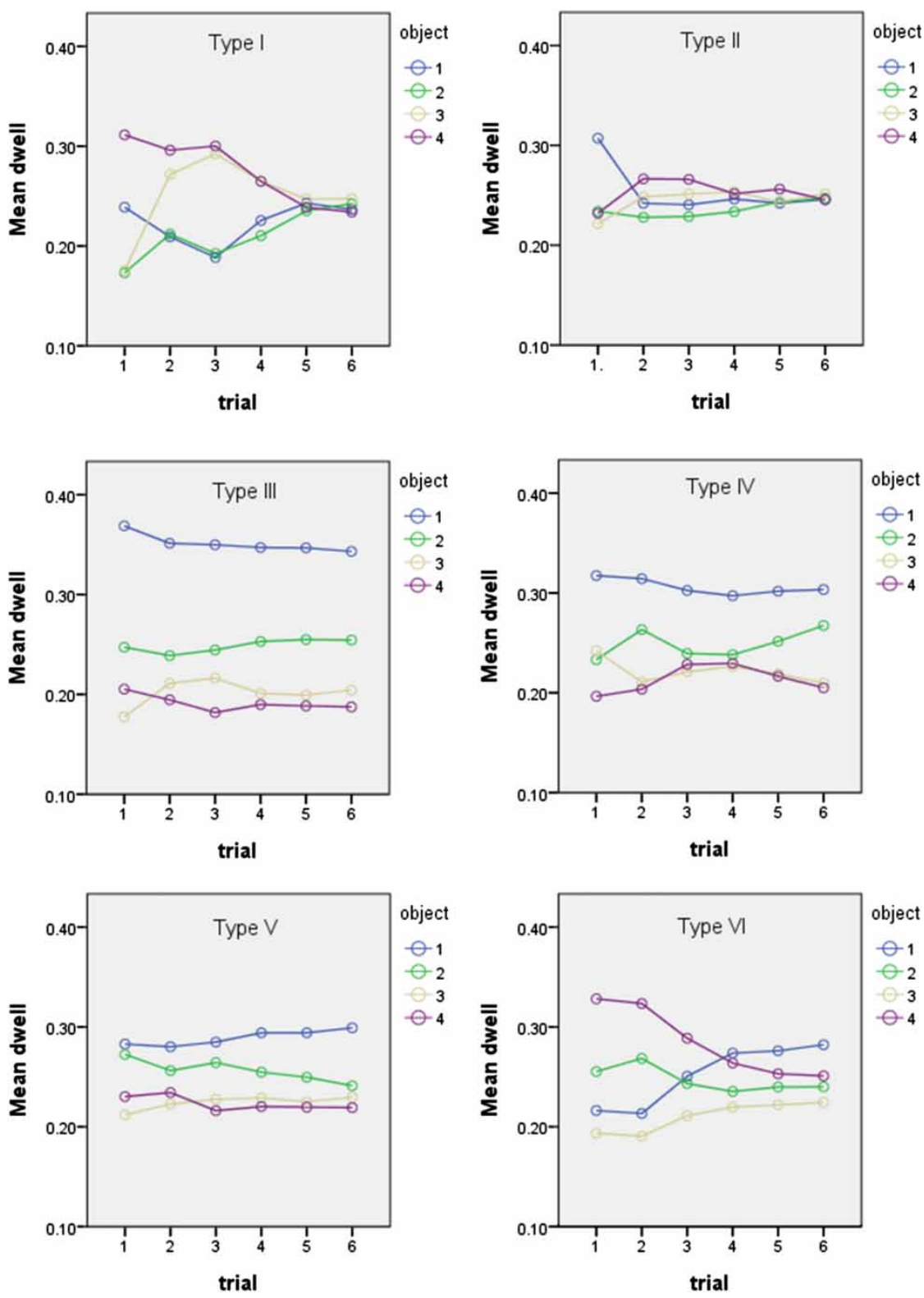

Figure A1. Moving averages. To view this figure in colour, please see the online issue of the Journal. 


\section{APPENDIX B: MULTILEVEL LOGISTIC REGRESSION}

In consequence of the fact that higher dwell time proportions for one object necessarily require lower dwell time proportions for other objects, these observations are not independent of one another. In other words, the dependent observations are nested within participants. Accordingly, in addition to the $t$-tests reported in the main text, multilevel logistic regression was used to evaluate the differences in looking times between objects of each type. Dwell time was predicted by repeated measure (across trials) in the first step and persons in the second step (in order to control between participants variation). When controlling for repeated measures and persons, we observe similar differences in dwell time proportions as was reported using the $t$-tests (see Tables B1-B3).

TABLE B1

Type III Multilevel logistic regression

\begin{tabular}{lllll}
\hline & \multicolumn{1}{c}{1} & \multicolumn{1}{c}{2} & \multicolumn{1}{c}{3} & 4 \\
\hline 1. Object 1 & - & - & - & - \\
2. Object 2 & $-.089^{* *}$ & - & - & - \\
3. Object 3 & $-.14^{* *}$ & $-.05^{* *}$ & - & - \\
4. Object 4 & $-.15^{* *}$ & $-.067^{* *}$ & $-.017(\mathrm{p}=.156)$ & \\
\hline
\end{tabular}

$* * p<.01$.

TABLE B2

Type IV Multilevel logistic regression

\begin{tabular}{lllll}
\hline & \multicolumn{1}{c}{1} & \multicolumn{1}{c}{2} & \multicolumn{1}{c}{3} & 4 \\
\hline 1. Object 1 & - & - & - & - \\
2. Object 2 & $-.058^{* *}$ & - & - & - \\
3. Object 3 & .036 & $-.094^{* *}$ & - & - \\
4. Object 4 & $-.06^{* *}$ & -.005 & $-.098^{* *}$ & \\
\hline
\end{tabular}

$* * p<.01$.

TABLE B3

Type $\vee$ Multilevel logistic regression

\begin{tabular}{lllll}
\hline & \multicolumn{1}{c}{1} & 2 & 3 & 4 \\
\hline 1. Object 1 & - & - & - & - \\
2. Object 2 & $-.058^{* *}$ & - & - & - \\
3. Object 3 & $-.07^{* *}$ & -.012 & - & - \\
4. Object 4 & $-.08^{* *}$ & -.022 & -.01 & \\
\hline
\end{tabular}

$$
* * p<.01 \text {. }
$$




\section{APPENDIX C: MANN-WHITNEY TESTS}

TABLE C1

Type I Mann-Whitney

\begin{tabular}{lllcl}
\hline & \multicolumn{1}{c}{1} & \multicolumn{1}{c}{2} & 3 & 4 \\
\hline 1. Object 1 & \multicolumn{1}{c}{-} & & \\
2. Object 2 & 22806 & - & - & \\
3. Object 3 & 22172.5 & 22707.5 & 24460 & - \\
4. Object 4 & 23293 & 23823 & \\
\hline
\end{tabular}

TABLE C2

Type II Mann-Whitney

\begin{tabular}{lcccc}
\hline & 1 & 2 & 3 & 4 \\
\hline 1. Object 1 & - & & & \\
2. Object 2 & 21987 & - & - & \\
3. Object 3 & 22718.5 & 24240.5 & 23046 & - \\
4. Object 4 & 22702.5 & 23925.5 & & \\
\hline
\end{tabular}

TABLE C3

Type III Mann-Whitney

\begin{tabular}{lllll}
\hline 1 & 2 & 3 & 4 \\
\hline
\end{tabular}

1. Object 1

2. Object 2

3. Object 3

4. Object 4

$* * p<.01$.

TABLE C4

Type IV Mann-Whitney

\begin{tabular}{lcccc}
\hline & 1 & 2 & 3 & 4 \\
\hline 1. Object 1 & - & & \\
2. Object 2 & 26616.5 & - & - & \\
3. Object 3 & $31856^{* *}$ & $28505^{* *}$ & 24031.5 & - \\
4. Object 4 & $32273.5^{* *}$ & $28879.5^{* *}$ & \\
\hline
\end{tabular}

$$
* * p<.01 \text {. }
$$


TABLE C5

Type V Mann-Whitney

\begin{tabular}{|c|c|c|c|c|}
\hline & 1 & 2 & 3 & 4 \\
\hline 1. Object 1 & - & & & \\
\hline 2. Object 2 & $27799.5^{* *}$ & - & & \\
\hline 3. Object 3 & $28809.5^{* *}$ & 23898.5 & - & \\
\hline 4. Object 4 & $29772^{* *}$ & 24799.5 & 23732.5 & - \\
\hline \multicolumn{5}{|l|}{$* * p<.01$} \\
\hline \multicolumn{5}{|c|}{$\begin{array}{c}\text { TABLE C6 } \\
\text { Type VI Mann-Whitney }\end{array}$} \\
\hline & 1 & 2 & 3 & 4 \\
\hline 1. Object 1 & - & & & \\
\hline 2. Object 2 & 25168 & - & & \\
\hline 3. Object 3 & $26470.5^{* *}$ & 23567.5 & - & \\
\hline 4. Object 4 & 25133 & 22350 & 20981.5 & - \\
\hline
\end{tabular}

${ }^{* *} p<.01$ 